WellBeing International

WBI Studies Repository

2015

\title{
The Use of Judgement Bias to Assess Welfare in Farm Livestock
}

L. Baciadonna

Queen Mary University of London

A. G. McElligott

Queen Mary University of London

Follow this and additional works at: https://www.wellbeingintlstudiesrepository.org/acwp_asie

Part of the Animal Studies Commons, Behavior and Ethology Commons, and the Comparative Psychology Commons

\section{Recommended Citation}

Baciadonna, L., \& McElligott, A. G. (2015). The use of judgement bias to assess welfare in farm livestock. Animal Welfare, 24: 81-91. doi: 10.7120/09627286.24.1.081

This material is brought to you for free and open access by WellBeing International. It has been accepted for inclusion by an authorized administrator of the WBI Studies Repository. For more information, please contact wbisr-info@wellbeingintl.org.

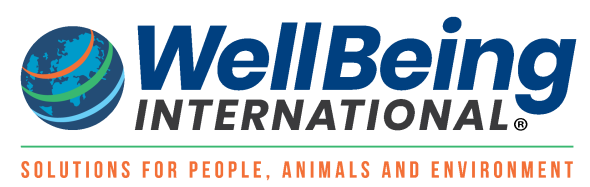




\title{
The Use of Judgement Bias to Assess Welfare in Farm Livestock
}

\author{
L. Baciadonna and A.G. McElligott \\ Queen Mary University of London
}

\section{$\underline{\text { KEYWORDS }}$}

affective state, animal cognition, animal welfare, emotions, optimistic-like, pessimistic-like

\begin{abstract}
The development of accurate measures of animal emotions is important for improving and promoting animal welfare. Cognitive bias indicates the effect of emotional states on cognitive processes, such as memory, attention, and judgement. Cognitive bias tests complement existing behavioural and physiological measures for assessing the valence of animal emotions indirectly. The judgement bias test has been used to assess emotional states in non-human animals; mainly in laboratory settings. The aim of this review is to summarise the findings on the use of the judgement bias test approach in assessing emotions in non-human animals, focusing in particular on farm livestock. The evidence suggests that it is possible to manipulate affective states and induce judgement bias effects in farm livestock. In addition, the results support the effectiveness of manipulating environmental variables for inducing negative or positive affective states. However, the evidence from farm livestock does not consistently confirm the directionality of the hypotheses. The use of specific strategies to induce positive or negative judgement bias, such as the manipulation of housing conditions, could account for the inconsistency of findings. The study of cognitive processes related to emotional states in livestock has great potential to advance and improve our understanding of animal welfare.
\end{abstract}

\section{Introduction}

The ability to experience affective states has a key role in the lives of animals (Panksepp 2005; Mendl et al 2009, 2010a; Briefer 2012). Emotions arise in salient situations and allow animals to maximise the acquisition of fitness-enhancing rewards and minimise the exposure to fitness-threatening cues (Rolls 2005; Burgdorf \& Panksepp 2006; Nettle \& Bateson 2012). Human psychology considers that emotional states have a multifaceted nature (Clore \& Ortony 2000; Lerner \& Keltner 2000). In addition to conscious experience of emotion, other components, such as behavioural and physiological changes associated with the emotional states are also included. For example, fear not only includes the subjective feeling of terror, but it is also associated with changes in heart rate, raised blood pressure and increased tendency for fleeing or freezing behaviour. While in humans linguistic reports are often used to investigate the conscious experience of emotion, the same approach cannot be used in animals. Instead, behavioural and physiological components are used to investigate emotional states in animals. Recently, Paul et al 
(2005) proposed to investigate the interactions between emotions and cognition. Cognitive processes and emotions interact in at least two possible ways: i) cognition can trigger particular emotional states; and ii) cognition can be influenced by specific emotional states (Dantzer 2002; Desiré et al 2002; Paul et al 2005). This is a crucial point due to the potential bidirectional causal relationship between cognition and emotion.

In humans, research has shown that cognitive processes, such as attention, memory and judgement are influenced by emotional states. The interaction between emotion and cognition has an adaptive value, as it helps to memorise information or make judgements about circumstances or stimuli (Mineka et al 1998; Paul et al 2005; Haselton \& Nettle 2006). Negative affective states, such as anxiety and depression, can cause increased attention and recall of threatening and negative stimuli (eg Eysenck et al 1991; Gotlib \& Krasnoperova 1998). Numerous experimental studies have been conducted on people with anxiety to examine attention biases, using mainly two computerized paradigms. The first is the visual dot probe task, in which two words are presented to participants on a computer screen and followed by a probe presented in the location of one of the two preceding words. The rationale behind this task is that participants experiencing negative affect will be quicker at detecting a probe when it replaces a threat word than when it replaces a neutral word (Paul et al 2005). The second paradigm is known as the Stroop colour-naming task, in which words are presented in a variety of colours and participants need to name the colour while ignoring the meaning of the word. These have shown that anxious subjects are particularly prone to bias their attention towards threatening information (Mathews \& MacLeod 1985, 1994; MacLeod et al 1986). Also, emotional states influence decisional processes, such as the likelihood of interpreting ambiguous information in a pessimistic or optimistic way (Johnson \& Tversky 1983; Wright \& Bower 1992; Nygren et al 1996). For example, anxious or depressed subjects tend to have pessimistic interpretations of ambiguous stimuli (Eysenck et al 1991). People in happy moods tend to overestimate the likelihood of positive outcomes and events, and underestimate the likelihood of negative ones instead (Wright \& Bower 1992; Nygren et al 1996). There is no reason to hypothesise that such effects are restricted to humans. In recent years, investigations of the cognitive components of emotions, together with other physiological and behavioural characteristics, have been regarded as a potential valuable source of information about animal emotions (Paul et al 2005; Mendl et al 2009).

A new integrative and functional theoretical approach has been proposed to assess emotions and mood in animals (Mendl et al 2010a). The conscious experience of emotions can be characterised in terms of valence and arousal defined as core affect (Russell 2003; Barrett et al 2007). Core affect is conceptualised in two dimensional axes and four different quadrants (Figure 1) in which emotional states are allocated: Q1) positive valence and high arousal; Q2) positive valence and low arousal; Q3) negative valence and low arousal; and finally Q4) negative valence and high arousal. The activity of the primitive bio-behavioural system that underpins the two evolutionary important functions of acquiring reward and avoiding punishment may map on to the Q3-Q1 and Q4-Q2 axes of the core affect space, respectively (Mendl et al 2010a). The core affect is a representation of subjective manifestation of any emotion or mood state and the space allows us to identify the structure of subjective emotional experience. This framework suggests that the measurement of different aspects of the emotional experience (eg neurophysiological, behavioural and cognitive components) is possible on two dimensions: arousal (intensity) and valence (negative or positive). The main advantages of this new approach are: i) to offer a structure to identify the wide range of emotional states in a functional perspective (ie according to the adaptive value of the emotional state); ii) to suggest how long-term mood state derives from short-term discrete emotions and how they might guide decision-making; iii) to generate novel measures of animal emotion and mood. The framework proposes that it is possible to impose (or remove) rewarding and punishing stimuli to generate specific affective states (Q1-Q4). This would allow making a priori 
predictions about how these will influence behaviours, physiological and cognitive read-outs of position in the core affect space, including judgement biases (Mendl et al 2010a).

Figure 1.

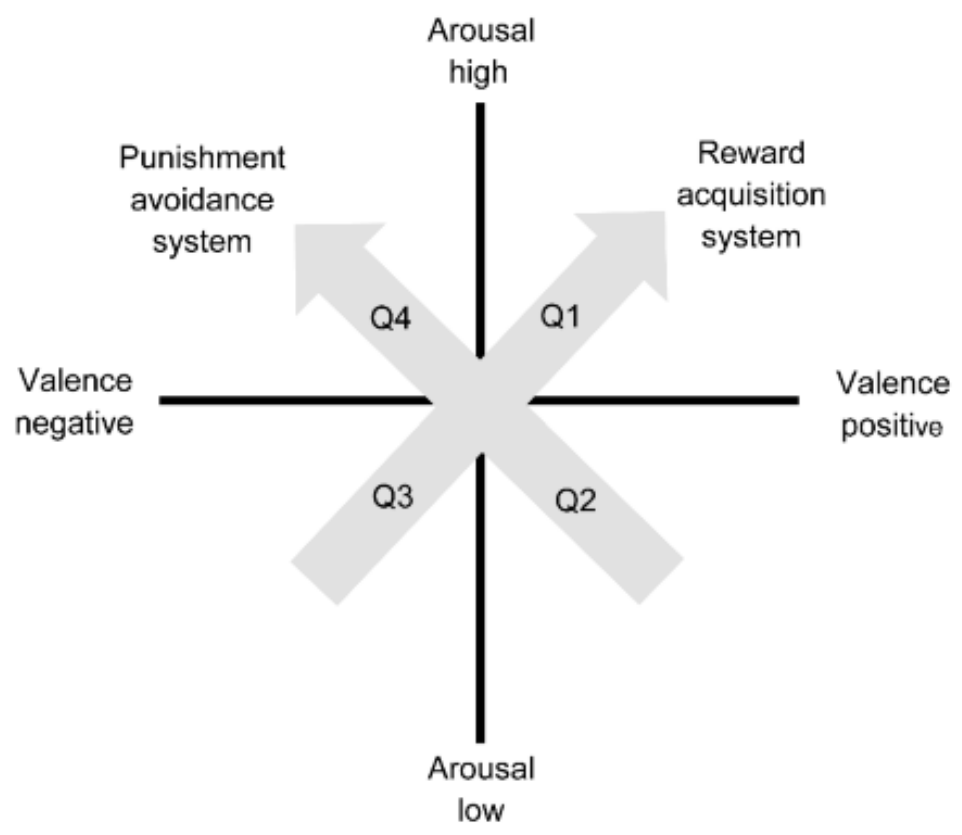

Core affect represented in a two-dimensional space. Positive affective states in quadrants Q1 and Q2, and negative affective states in quadrants Q3 and Q4. Arrows indicate putative biological and behavioural system associated with reward (Q3 and Q1) and avoidance (Q2 and Q4). Adapted from 'An integrative and functional framework for the study of animal emotion and mood', by Mendl M, Burman OHP and Paul ES 2010a Proceedings of the Royal Society B277: 2895-2904. Copyright 2010 by the Royal Society. Adapted with permission.

The first study exploring the association between induced emotional states and cognitive bias in animals was by Harding et al (2004). The research consisted of a training phase in which rats (Rattus norvegicus) had to discriminate between two sound stimuli at different frequencies ( 2 or $4 \mathrm{kHz}$ ), signalling a positive event (food) or a negative event (no food and noise). Rats had to perform a particular operant response (ie pressing a lever) to obtain the food or refrain from pressing a lever to avoid unpleasant white noise. Once trained on this task, rats were allocated to either predictable or unpredictable housing. After the housing manipulation, the rats were tested with non-rewarding probe tones of intermediate frequency $(2.5,3.0$ and $3.5 \mathrm{kHz})$. The hypothesis was that rats experiencing negative emotional states (housed in unpredictable conditions) would be more prone to judge ambiguous tones as predicting negative events, while rats in positive emotional states would show an opposing trend. Rats under unpredictable housing condition behaved as expected, making a lower proportion of positive responses (pressing a lever) when the ambiguous tone was close to the tone positively associated with the food as well as with the food tone itself. They were also slower in making these responses compared with the control condition.

Since Harding et al (2004), others have tested cognitive bias in a diverse array of animals. These include studies on dogs (Canis lupus familiaris; Mendl et al 2010b; Burman et al 2011), starlings (Sturnus 
vulgaris; Bateson \& Matheson 2007), and rats; Burman et al 2008a, 2008b, 2009; Matheson et al 2008), which have all replicated the findings, and confirmed the link between emotional states and cognitive processes. However, the findings have not been supported in some other studies involving starlings (Brilot et al 2009), hens (Gallus gallus; Wichman et al 2012) and bears (Ursus arctos horribilis; Keen et al 2013). Nevertheless, overall, the wide variety of species used and the range of experimental contexts in which judgement bias has been tested provide a good indication of the external validity of the task and support the hypothesis that it reflects emotional states.

Studies investigating the link between emotional states and cognitive processes have been reviewed by Mendl et al (2009). This review summarised studies from 2004 to 2009 and includes published papers or conference abstracts, as well as unpublished findings and two human studies. In the review, the authors discuss the generality of findings, and comment on the influence of feeding motivation, general activity and learning on the results. Also, they discuss whether the bias observed, the manipulation adopted and the set-up of the task could provide information about different types of affective states. Most of the studies considered provided evidence for judgement bias, confirming the predictions from the original Harding et al (2004) study. Where the results were not in line with predictions, reasons such as the use of a wide range of species and different affect manipulation paradigms were suggested.

\section{Judgement bias and farm livestock}

The aim of our review is to extend Mendl et al's (2009) work, and focus specifically on farm livestock in this fast-moving and important area of animal welfare research. Indeed, 25 papers on cognitive bias have been published during the period from the Mendl et al (2009) review until May 2014, and 14 of them have focused on farm livestock. In particular, more recent studies investigated the role of experimentally induced emotional states on cognitive processes. Interest in this area of research is increasing greatly and could guide potential applications to improve animal well-being. The welfare of animals, including physical and mental well-being, is a major concern for society (Duncan 1996; Dawkins 2006, 2008; Wathes 2010). The recently suggested idea of mental well-being implies that animals are sentient (ie have/express emotions) and are responsive to the environment (Boissy \& Erhard 2014). One of the aims of welfare science is to provide experience of a "life worth living" (FAWC 2013). This is just one example of how the focus of attention has changed from simply avoiding neglect and suffering, to providing and promoting positive welfare (Boissy et al 2007). In particular, understanding how physical and psychological distress causes negative emotional states (both acute or chronic stress) that lead to longer term moods is vitally important (defined as sum of short-term emotional episodes; Nettle \& Bateson 2012). The judgment bias test represents an innovative, versatile and feasible way to investigate emotional states in farm livestock.

In this review we first describe the methodology and criteria used for the selection of studies included. Then, we illustrate the main paradigms used to study the judgement bias test, highlighting strengths and weaknesses. Finally, we report the main findings of the studies selected. In the last sections of this review we provide a summary of findings and discuss potential limitations and future directions.

\section{Literature search and study selection}

For inclusion in our review, we used the following criteria for studies: i) published in peer-reviewed journals; ii) English language; iii) experimental studies of animal subjects; and iv) use of pharmacological treatment to induce emotional states. The electronic databases Ovid, Pubmed and Web of Knowledge were used to identify the relevant papers and no temporal limits were used. PRISMA guidelines were used to conduct the literature search (Moher et al 2009). The keywords used to conduct the search were: 'Animal' and ('Welfare' or 'Mood' or 'Emotion') and 'Cognitive bias'. The authors (LB and AG) were 
responsible for the literature search, final screening and assessment for eligibility. Criteria compliance was agreed by two authors. Bibliographies from all relevant reviews were inspected for additional studies not yielded by the search. A total of 249 papers were identified; and 32 papers were included after checking for key criteria and removing duplicates. The main characteristics of the 32 studies included in the review are reported in Tables 1 and 2 (see supplementary material to papers published in Animal Welfare on the UFAW website: www.ufaw.org.uk). The experimental paradigms and main findings will be described.

\section{Experimental paradigms}

Three different paradigms were used in the selected studies: i) go/no-go task; ii) active choice task; and iii) natural behaviour task.

\section{Go/no-go task}

This task was used in the first study (Harding et al 2004) and has formed the basis for most subsequent research. In this task, animals are trained to perform a response associated with a cue (auditory, visual, spatial and olfactory) in order to experience a positive event (eg food), and to perform a different response to avoid a negative or less positive event. After training, animals are presented with an unreinforced ambiguous cue. According to the human literature (for a review on humans and animals, see Paul et al 2005), subjects in a putative negative emotional state would be more likely to categorise the ambiguous cue as predicting the negative event and thus more likely to show the negative response (ie negative judgement bias).

The majority of the studies $(\mathrm{n}=22)$ included in this review used the go/no-go task paradigm (Harding et al 2004; Bateson \& Matheson 2007; Burman et al 2008a, 2011; Brilot et al 2009; Doyle et al 2010, 2011a,b; Mendl et al 2010b; Bateson et al 2011; Sanger et al 2011; Boleij et al 2012; Destrez et al 2012, 2013; Douglas et al 2012; Richter et al 2012; Wichman et al 2012; Briefer \& McElligott 2013; Neave et al 2013; Daros et al 2014; Verbeek et al 2014a,b) and a judgement bias was found in 20 studies. However, some concerns linked to this task have been suggested (Mendl et al 2009). In particular, in its current form, it does not allow disentangling whether the responses emitted are due to low motivation in completing the task or an effective negative judgment bias. However, a lowered response to the positive stimulus might indicate the effect of low motivation in completing the task. Another potential limitation is that it is not possible to exclude that the bias found is due to the repeated number of trials used during the training phase (ie learning process).

\section{Active choice task}

The active choice task for studying judgement bias was developed by Matheson et al (2008) and requires the subject to respond actively to both the positive and negative stimuli. In other words, the subject needs to make the same type of response to both cues (eg press right lever vs press left lever; dig in right bowl vs dig in left bowl). Subjects are always reinforced with food. The necessary differential value in the reinforcements is generated by delaying or decreasing the reward (ie the positive stimulus is associated with immediate reward, whereas the negative stimulus is associated with delayed reward or with a reduced amount of food), or by presenting an aversive stimulus (Rygula et al 2012; Papciak et al 2013). This task has been used in seven studies included in this review (Matheson et al 2008; Brilot et al 2010; Brydges et al 2012; Pomerantz et al 2012; Rygula et al 2012; Keen et al 2013; Papciak et al 2013) and a judgement bias has been found in six of them. The active choice task allows the limitations of the go/nogo task to be overcome, in that it does not allow omission responses which might be due to pre-existing motivational states or temperamental traits (eg low levels of novelty seeking and impulsivity) (Brilot et al 
2010; Papciak et al 2013). In other words, the advantage is that by using the same type of response (eg lever press) for both cues, any general changes in motivation to show this response that are induced by affect manipulations apply equally to the two training cues. The disadvantage of this task is that it requires several training sessions.

\section{Natural behaviour tasks and alternative task}

Natural behaviour tasks are based on animals' spontaneous responses of approaching/avoiding specific cues. The use of this task is relatively recent and only two studies included in this review have used it (Brilot et al 2009; Salmeto et al 2011). In the first experiment (Brilot et al 2009), starlings were tested on their approach or avoidance response to food close to aversive eye-spot stimuli. The stimuli were presented in either an unambiguous or ambiguous form and the main hypothesis was that birds in more negative affective states would be more likely to delay their approach to ambiguous stimuli. The hypothesis was not supported. The authors suggested that the affect manipulation strategy might have not been effective in inducing an emotional response.

In the second study (Salmeto et al 2011), chicks were exposed to two different conditions: 5 min of isolation to induce a putative anxiety-like state and $60 \mathrm{~min}$ of isolation to induce a depressive-like state. They were then tested in a straight alley maze with a series of morphed ambiguous potentially attractive chick silhouette cues and aversive owl silhouette cues. The results showed that in the control group (nonisolated chicks), runway start and goal latencies generally increased on the basis of the aversive characteristics of cues. In chicks in the anxiety-like state, runway latencies increased for aversive ambiguous cues, reflecting more pessimistic-like behaviour. In chicks in the depression-like state, runway latencies increased for both aversive and appetitive ambiguous cues, reflecting more pessimistic-like overall. Natural behaviour tasks have the potential to not require training. This is in contrast to protocols that use visual or auditory cues, which require large numbers of conditioning trials. However, the use of appetitive and aversive cues eliciting spontaneous approach and avoidance behaviour is effective only when a salient cue is selected (Brilot et al 2009) and further investigation is needed to clarify the nature of the specific decision-making processes that this paradigm measures.

One study used an alternative task to test judgement biases in rats (Burman et al 2008b). In this experiment, rats were trained to run down a runway for 12 pellets of food (Burman et al 2008b). The size of the food reward was decreased to just one pellet for all remaining trials once the rats started to run at a constant speed. Decreasing the amount of food reward for which the rats had been trained, increased the sensitivity to reward loss and induced a negative emotional state. Burman et al (2008b) found that rats ran more slowly for the smaller reward than those that had been trained to run for one pellet throughout the study. Also, rats that had been switched from enriched to barren housing showed a longer lasting negative contrast effect than those that remained in the enriched environment and received additional enrichment objects. The conclusion was that animals in the negative affective state appeared to be more sensitive to loss or failure.

\section{General findings}

The studies summarised in Table 1 (www.ufaw.org.uk) were carried out on six mammalian species, two bird species and one insect species. Cues of five different types (auditory, visual, spatial, tactile and olfactory) and a variety of experimental manipulations to induce affective emotions were employed. The literature synthesis shows that 29 studies found evidence for judgement bias following emotional manipulation (see Tables 1 and 2). Three studies did not find a bias and reported methodological explanations to account for this (Brilot et al 2009; Wichman et al 2012; Keen et al 2013). Brilot et al (2009) proposed that the use of eyespots in their experiment might have not been effective to induce fear 
and anxiety due to the lack of resemblance with any biologically relevant stimuli. Similarly, the enriched environments used might have not been effective to induce a change in the emotional states because of the limited time of exposure (Keen et al 2013) or too small differences between the basic and the enriched environment (Wichman et al 2012). These findings show that the effective induction of putative emotional states might have critical importance in the assessment of judgement bias.

\section{Livestock animals}

\section{Studies inducing acute and chronic stress}

Ten studies investigated the effect of a stressor on judgement bias in livestock. The first paper (Doyle et al 2010) found that restrained and isolated sheep (RIS) had a more positive interpretation bias than control subjects. These results were in contrast to the a priori hypothesis that the RIS condition would induce negative judgment bias. It is possible that the effects of the RIS procedure were no longer evident when the sheep were tested on the judgment bias test. Also, sheep might have been prone to seek a positive event to balance their situation following a negative event experience (Spruijt et al 2001). In a second study from the same research group, lambs exposed to unpredictable, aversive events over a longer period of time (three weeks) were found to show negative judgement (Doyle et al 2011a). The authors suggested that the results could be due to a pessimistic-like judgement bias, but it is also possible that the lambs learned that ambiguous locations were unreinforced and subsequently showed less approaches to ambiguous locations. The stressed lambs learned more rapidly than control lambs that the ambiguous locations were unreinforced. However, in this experiment the stressed lambs under aversive and unpredictable events for four weeks (eg restrained, inaccessible food) did not show physiological evidence of a chronic stress, which posits the question on whether and to what extent the animals became effectively distressed.

In a similar experiment, Sanger et al (2011) investigated the effect of release from the acute stress of shearing on judgement bias in sheep. Twenty-four sheep (Ovis aries) were tested individually and divided in two cohorts ( $n=6$ control, and $n=6$ shorn animals) following the shearing procedure on two consecutive days. Both cohorts were tested again after eight days. It was found that releasing sheep from the acute stress of shearing produced a positive judgement bias in the first cohort of animals, in line with what was previously found by Doyle et al $(2010,2011$ a) and Spruijt et al (2001). However, the results were not replicated in the second cohort of animals. The control group of the second cohort was tested the day after seeing and hearing the shearing procedure. The shearing procedure, in fact, took place outside and close to experimental sheep. This might have induced anticipatory anxiety followed by a positive judgement bias, and cancelled the effect of treatment between groups. The results of the first cohort seem more reliable as they are not affected by this methodological issue. Non-significant results were obtained when both cohorts were re-tested after eight days. This could indicate a rapid recovery from an acute stressor.

Destrez et al (2012) investigated the effect of chronic stress on the judgement bias test in sheep. In contrast to Doyle et al (2011a), chronic stress was provided for an extensive period of nine weeks in which lambs were under unpredictably and uncontrollably subjected to negative events in a farm setting (presence of dog, odour of killed conspecific, and human handling procedure). The group exposed to prolonged chronic stress had a negative judgement bias for all the ambiguous cues and the negative cue, compared with the control group. The treated group also took longer to approach the location of cues. Lambs were tested for two consecutive days and the responses of the treated group were more salient on the second day. 
Verbeek et al (2014a) showed that food restriction not only influenced judgment bias, but also attention toward food-related stimuli in sheep. In this study, two groups were treated differently in terms of food availability. The high feeding level group received gradually increasing quantities of food during seven days (ie from 110 to $170 \%$ of maintenance required); whereas the low feeding level group received decreasing amounts of food (from 58 to $50 \%$, and again to $58 \%$ of maintenance required). The study showed that the low feeding level group had more optimistic judgement bias despite the decreased amount of food received and the resulting weight loss. One possible explanation is that a short period (seven days) of food restriction may not have been enough to induce a negative affective state. The fact that restricted animals approached and judged optimistically ambiguous cues confirms this explanation. Food restriction could have activated exploratory behaviours and locomotor activity associated with hunger. Also, hungry sheep could have been more incentivised to take some risks to find food. Overall, the results of the study showed that food restriction altered the behaviour of sheep (activate the animal in order to find food), but further investigations are needed to clarify the mechanisms through which the change happened.

Neave et al (2013) investigated whether the dehorning procedure of dairy cattle calves (Bos taurus) produced changes in emotional states that would be evident in a judgement bias task. The main hypothesis was that calves in pain after dehorning would show a pessimistic bias in judging the ambiguous stimuli. Calves were tested on a touch screen go/no-go task twice. In the first experiment, they were tested 2, 16 and $26 \mathrm{~h}$ before being sedated for the dehorning procedure and 6 and $22 \mathrm{~h}$ afterwards. In the second experiment, the calves were tested 2 and $16 \mathrm{~h}$ before and 6 and $22 \mathrm{~h}$ afterwards. The study showed that animals before the dehorning procedure approached the ambiguous stimuli with a similar proportion of that observed in the training phase. After the dehorning procedure, calves approached the ambiguous stimuli less, showing a pessimistic bias. Overall, calves experienced a negative emotional state for at least $22 \mathrm{~h}$ after dehorning.

Using similar methodology, Daros et al (2014) investigated the effect of separation from their mothers on calves using the judgement bias test. They then subsequently compared this effect with that of the dehorning procedure. Animals were tested at baseline, after the separation, and 12, 36 and $60 \mathrm{~h}$ later. The results showed a negative judgement bias of calves (reduction of 'go' responses) after $36 \mathrm{~h}$ of separation from the mother, which was similar to the bias found after dehorning. This finding is particularly interesting as it demonstrates how psychological and physical stressors might have the same effect on emotions and cognitive processes.

Briefer and McElligott (2013) investigated the impact of past experience of poor welfare (rather than short-term distress), on decision-making in goats (Capra hircus). The study compared a group with a history of poor welfare with a control group that had experience of good welfare. The authors applied Codes of Recommendation for the Welfare of Goat (DEFRA 1989) on the distinction between good or poor welfare. The study was aimed at investigating any differences in mood (long-term emotional states not linked directly to the event) after the animals had received more than two years of good care. The hypothesis was that prior poor welfare conditions could have induced a negative long-term effect on the goats' mood. The study found no overall effect of past welfare conditions during a judgement test. This finding indicates that goats could recover from the effects of long negative experiences. Also, results highlight the crucial role of prolonged good care experiences to reduce the impact of negative experiences on a judgement bias test. Briefer and McElligott (2013) found sex differences in the interaction between welfare experience and cognitive bias; the female group that had experienced poor welfare had an optimistic bias compared to females in the control group. The results showed no difference between the poor welfare and control groups in male goats. 
Although all the above studies investigated the effect of a stressor on cognitive bias in livestock species, the time of exposure to the stressful event varied amongst them. In particular, some studies (Doyle et al 2010; Sanger et al 2011; Verbeek et al 2014a) used an acute stressor (3 min per week), whereas others (Doyle et al 2011a; Destrez et al 2012) employed a chronic stressor lasting 3 or 4 weeks. Contrary to their hypotheses, the first group of studies found a positive judgement bias following exposure to acute stressor. Studies using exposure to chronic stress, instead, confirmed the induction of a negative judgement bias. One possible explanation for these findings is that releasing animals from short-term exposure to stressors could induce stronger emotional effects (ie positive) than the experimental manipulation (ie negative). By contrast, exposure to chronic stress could induce longer term negative emotions (ie negative mood) and a pessimistic bias. However, when interpreting findings, it is important to take into account not only the duration of the treatment, but also the duration of the stress experienced by the animal. It is plausible that different forms of short-term treatment might have different effects on emotional states. Some treatments might not generate emotional state change whereas others (even when acute), might have long-lasting effects, with an ongoing presence during testing.

Other variables that might affect results across studies are, the duration of the training phase and the outcome of task learning. For example, the majority of studies have used a cut-off of 25 to $30 \mathrm{~s}$ for two consecutive training sessions to define the learning of the 'no go' response. The cut-off for the 'go' (approach) response was usually less than $10 \mathrm{~s}$. After this phase, animals were tested. Briefer and McElligott (2013) did not include a specific target duration for the go/no-go responses, but used instead the significant difference between approach/non-approaches as an outcome measure of successful training. Surprisingly, a positive bias was found in response to the 'negative' stimulus (ie stimulus learned to be negative during the training), although no experimental manipulations had been administered between the training and the testing phase. A possible interpretation for these findings is that poor animal welfare might affect learning times (eg longer), as well as the performance on the judgement bias task.

\section{Studies using environmental enrichment}

Two studies have investigated the impact of housing conditions on decision-making (Douglas et al 2012; Wichman et al 2012). Douglas et al (2012) tested pigs (Sus scrofa) housed in two different housing conditions (enriched vs barren) in four consecutive tests alternating the housing condition in the animal group over the experiment. According to their hypothesis, pigs housed for five weeks in an enriched environment were more likely to respond positively to an ambiguous auditory cue than pigs housed in a barren environment. In addition, the study explored the performance on the judgement bias test when subjects had been allocated to the other housing condition (from barren to enriched and vice versa) 2-7 days before being tested. Then, they were moved to the original condition (enriched to barren) and retested two and seven days afterwards. This complex design had the purpose of testing any interactions between the different environments and the judgment bias. Animals kept in the enriched environment approached the ambiguous stimuli more often (more optimistic bias) than the animals kept in the barren environment, independently of their training environment. Pigs trained in the enriched housing condition were more pessimistic when moved to the barren housing condition. Animals with prolonged experience (five weeks) of the enriched environment were more sensitive to a reduction in the quality of the environment than those that had experienced the same condition during a shorter period (seven days). This study confirms the impact of changes in housing conditions on judgement bias (Bateson \& Matheson 2007). This study is the only one that applied a design based on auditory rather than visual discrimination. However, after realising that pigs were unable to discriminate a glockenspiel sound that was an octave different from the positive and negative cues, the researchers introduced a change in the procedure. They decided to use three different sounds to anticipate the negative, positive and ambiguous cues (ie they used a clicker, the glockenspiel, and a dog toy sound, respectively). 
Wichman et al (2012) did not find clear evidence for the effect of housing conditions on domestic chicks. In this experiment, chicks were tested twice with a cross-over design. The hypothesis was that chicks in the enrichment housing condition would be in a positive affective state, and therefore faster to approach the ambiguous cue. Contrary to the hypothesis, the chicks in the enriched condition had a tendency to approach the middle cue more slowly. The authors suggested that this tendency could be attributed to the small differences between the two experimental conditions in terms of the enrichment provided. Furthermore, the extra food provided as part of the enrichment condition may have reduced the motivation of the chicks to work for the reward. Correlational analyses highlighted that other factors, such as individual fear level, and relationship between chicks and motivation to feed could influence the performance on the cognitive bias test and explain the unexpected results. Almost all the studies that have investigated the effect of housing conditions on cognitive bias (Bateson \& Matheson 2007; Douglas et al 2012), used a set-up of good or poor housing (enriched or not) with a prediction that animals in poor housing conditions would express negative responses to ambiguous stimuli. It has been more difficult to find evidence of positive judgement bias in animals that had a temporal transition from standard to enriched housing condition, with the exception of a few (Burman et al 2009; Doyle et al 2010).

Overall, the research supports the evidence of using judgement bias tasks to assess emotional states in livestock, and the effectiveness of manipulating environmental variables, such as enrichment and welfare practices to induce negative or positive emotional states. However, the use of different species, protocols, and methodologies limit the possibility for comparing findings and drawing definitive conclusions. The use of multi-dimensional measures of emotional states (ie physiological as well as behavioural parameters; Paul et al 2005; Boissy et al 2007) would facilitate much better interpretation of the findings of future studies.

\section{Pharmacological treatment in farm livestock}

Other strategies to induce changes in emotional states include using pharmacological treatments. To date, this approach has been applied only in sheep (Doyle et al 2011b; Destrez et al 2013; Verbeek et al 2014b). The administration of a serotonin-antagonist (p-Chlorophenylalanine [pCPA]) in a group of 15 sheep, for example, was associated with a pessimistic response during the judgement bias task (Doyle et al 2011b). The experimental design used in this study (Doyle et al 2011b) included two groups of animals (controls, which received a saline injection, and treated, which received the injection of $40 \mathrm{mg} \mathrm{kg}-1$ of pCPA). Sheep were tested on the judgment bias test after three and five days of pharmacological treatment, and five days after the cessation of treatment. The effect of pCPA was visible after five days (ie the treatment group approached the ambiguous location less than the control group showing a negative judgement bias) and a trend (negative judgment bias) was found after the cessation of the treatment. No effects were observed during the three days of treatment. Overall, the serotonin-antagonist (which is involved in causing negative emotional symptoms) decreased the level of serotonin in the brain and induced depression-like behaviours.

(Destrez et al 2013) investigated the use of diazepam to reduce negative bias of ambiguous stimuli. This hypothesis was based on the evidence that benzodiazepine has an effect on the reduction of negative affective states, such as anxiety and fear in cattle (Sandem et al 2006). A sample of 20 lambs was tested twice using a spatial differentiation test (Burman et al 2008; Doyle et al 2010), 10 min and $3 \mathrm{~h}$ after the injection of diazepam. The control group took longer to get closer to the positive ambiguous location than the treated group. This finding was interpreted to suggest that the treated group showed a positive judgement bias due to fear reduction, associated with the administration of diazepam. In the same study (Destrez et al 2013), the treated group also showed a reduction of fear in isolation and during a suddenness test. It is possible that the injection of diazepam may have induced a reduction of mnemonic capacity to differentiate the positive and close to positive locations. 
Verbeek et al (2014b) investigated how morphine (an opioid agonist) and naloxone (opioid antagonist) affect judgement bias after receiving two different rewards in sheep. The hypothesis was that consuming palatable food would generate a more optimistic bias, and that the injection of morphine would boost this bias and reduce the effect of unpalatable reward (ie pessimistic bias). It was also hypothesized that naloxone would generate opposing results, preventing the optimistic bias after palatable food and producing a small effect on unpalatable food. The results were to some extent in line with predictions, except for the naloxone which did not affect the judgement bias test and thus did not induce a different affective state in sheep. However, the results were based only on a single session during one day even though animals were tested twice. Verbeek et al (2013) also investigated the effect of administration of ghrelin in sheep, a peptide involved in the regulation of behavioural adaptations to food intake regulation. In this study, administration of ghrelin induced a pessimistic judgement bias (ie increased motor activity).

The use of pharmacological treatments to induce positive and negative emotional states has the potential to clarify the mechanisms behind the formation of pessimistic and optimistic bias in the judgment of ambiguous stimuli. However, the interpretation of results is difficult. For example, the role of serotonin depletion on learning capacities is controversial, as there is evidence for both reduction of learning (ie short-term memory capacities which are involved in the judgement bias paradigm) as well as no effect on learning (Verbeek et al 2014b). In Doyle et al (2011b), the control group and the pCPA group learned at different rates that ambiguous stimuli were not reinforced, supporting the first hypothesis. Depletion of serotonin also reduced reactivity in sheep (Doyle et al 2011b) as measured by lower rates of vocalisations when animals were separated from the flock (isolation test) compared with the control group. However, the reduction of reactivity was not supported in a task involving exploration of a novel object. In fact, in this task, the treated group approached the unknown objects more often. These studies suggest that serotonin could affect behaviours in two different ways. Namely, it could induce depressionlike symptoms, as well as fear/anxiety states, which could explain the reduction of reactivity highlighted in an isolation test but not in the novel object test.

\section{Discussion}

\section{Summary of findings}

The aim of this review is to summarise and discuss studies using cognitive bias methodology to assess emotional states in animals. In particular, the research included in this review aimed to test the hypothesis that inducing a putative emotional state has a temporary effect on information processing (ie judgement of an ambiguous stimulus). The summary of these studies indicates that $28 / 32$ studies found a judgement bias. However, the predictions related to the valence of the bias were not always confirmed. This raises the interesting question as to whether the intended emotional states were successfully induced and tested. A multimodal assessment (Briefer et al 2015) of the emotional state induced prior to judgement bias testing might provide an answer to that question, and a stronger rationale for interpreting the successful induction of a judgement bias. Results from five studies in livestock species indicate that animals exposed to long-term stressors (Doyle et al 2011a; Destrez et al 2012), psychological stress (Daros et al 2014) or receiving specific pharmacological treatments (ie pCPA and ghrelin; Doyle et al 2011b; Verbeek et al 2014b) have a negative judgement bias. By contrast, four studies show the presence of a positive judgement bias, mainly by including changes to housing conditions (Douglas et al 2012), routine care (Briefer \& McElligott 2013), and using diazepam and morphine (Destrez et al 2012; Verbeek et al 2014b). Surprisingly, releasing animals from short-term stressors induced positive emotional states (Doyle et al 2010; Sanger et al 2011; Verbeek et al 2014a), with the exception of one study (Neave et al 2013). However, in Neave et al (2013), it might be that calves were still experiencing the pain from the dehorning at the time of testing. This indicates that different forms of short-term 
treatment might have different effects on emotional states. Some might not generate emotional state change, whereas others might have long-lasting effects with an ongoing presence during testing.

Overall, the research findings support the use of judgement bias tests to explore emotional experiences in animals. The possibility of successfully testing emotional states in animals is particularly relevant in farm settings. Indeed, one of the aims of welfare practices is to promote a better quality of life in livestock (Dantzer 2002; Paul et al 2005; Boissy et al 2007; Mendl et al 2010a; Wathes 2010; FAWC 2013). The use of cognitive bias tasks could inform the validity and implementation of strategies to increase positive moods and decrease stress in farm livestock.

\section{Limitations}

Almost all the studies included in this review used the go/no go task. However, the go/no go task, does not allow us to clearly disentangle the effects of training from those of animals' pre-existing motivations, and requires several sessions of training. The use of different paradigms, such as those based on active choices and natural behaviours might help overcoming these limitations. Across all these paradigms, the assessment of the rewarding and punishing properties of the stimuli and the assessment of animals' cognitive abilities to discriminate between those (eg exact quantity of food needed in order to perceive it as positive or negative) appear to be crucial to draw significant and reliable conclusions on the effect of emotions on cognitive bias. Similarly, the assessment of animals' cognitive and sensory abilities to discriminate positive, negative, and ambiguous cues and the differences between them might improve the reliability of findings. Finally, the evaluation of species-specific differences and individual personality characteristics could help our understanding of baseline differences in animals' motivation to approach/avoid rewarding and punishing stimuli, which might affect the performance on the judgement bias task. One of the limitations of this review is that only a minority of studies were explicitly aimed at investigating positive emotions using the judgment bias task. This might indicate that the identification of rewarding stimuli is more difficult than the identification of negative stimuli. Establishing what constitutes a positive experience for animals has the potential to inform the use of the judgment bias test to test the effect of positive emotional states on cognitive processes.

\section{Future directions}

Boissy et al $(2007,2014)$ suggest that cognitive, rather than environmental, enrichment could be a viable option to induce positive emotional states. The use of physiological and vocal measures complementing the identification of behavioural approach and avoidance could also strengthen and clarify the outcomes of cognitive bias tasks and provide information on emotional arousal as well as valence. Finally, the use of computational models could help identify and assess cognitive and motivational variables which might affect the performance on the judgment bias test (Trimmer et al 2013).

\section{Conclusion}

This review supports the use of judgment bias tasks to assess negative emotional states in animals. The use of this task to assess positive emotional states has not been explored extensively yet, but has the potential to inform welfare practices in livestock. The assessment of animal personality differences and cognitive-sensory abilities, and the identification of emotionally salient cues could improve the understanding and reliability of the findings obtained from using cognitive bias paradigms. 


\section{Acknowledgements}

We thank Magda Osman, the editor and two anonymous reviewers for very helpful comments.

\section{References}

Barrett LF, Mesquita B, Ochsner KN and Gross JJ 2007 The experience of emotion. Annual Review of Psychology 58: 373-403. http://dx.doi.org/10.1146/annurev.psych.58.110405.085709

Bateson M and Matheson SM 2007 Performance on a categorisation task suggests that removal of environmental enrichment induces 'pessimism' in captive European starlings (Sturnus vulgaris). Animal Welfare 16: 33-36

Bateson M, Desiré S, Gartside SE and Wright GA 2011 Agitated honeybees exhibit pessimistic cognitive biases. Current Biology 21: 1070-1073. http://dx.doi.org/10.1016/j.cub.2011.05.017

Boissy A and Erhard HW 2014 How studying interaction between animal emotion, cognition, and personality can contribute to improve farm animal welfare. In: Grandin T and Deesing JM (ed) Genetics and the Behavior of Domestic Animals pp 81-113. Academic Press: CA, USA. http://dx.doi.org/10.1016/B978-0-12-394586-0.00003-2

Boissy A, Manteuffel G, Jensen MB, Moe RO, Spruijt B, Keeling LJ, Winckler C, Forkman B, Dimitrov I, Langbein J, Bakken, M, Veissier I and Aubert A 2007 Assessment of positive emotions in animals to improve their welfare. Physiology \& Behavior 92: 375-397. http://dx.doi.org/10.1016/j.physbeh.2007.02.003

Boleij H, van't Klooster J, Lavrijsen M, Kirchhoff S, Arndt SS and OhI F 2012 A test to identify judgement bias in mice. Behavioural Brain Research 233: 45-54. http://dx.doi.org/10.1016/j.bbr.2012.04.039

Briefer EF 2012 Vocal expression of emotions in mammals: mechanisms of production and evidence. Journal of Zoology 288: 1-20. http://dx.doi.org/10.1111/j.1469-7998.2012.00920.x

Briefer EF and McElligott AG 2013 Rescued goats at a sanctuary display positive mood after former neglect. Applied Animal Behaviour Science 145: 45-55. http://dx.doi.org/10.1016/j.applanim.2013.03.007

Briefer EF, Tettamanti F and McElligott AG 2015 Emotions in goats: mapping physiological, behavioural and vocal profiles. Animal Behaviour 99: 131-143. http://dx.doi.org/10.1016/j.anbehav.2014.11.002

Brilot BO, Asher L and Bateson M 2010 Stereotyping starlings are more "pessimistic". Animal Cognition 13: 721-731. http://dx.doi.org/10.1007/s10071-010-0323-z

Brilot BO, Normandale CL, Parkin A and Bateson M 2009 Can we use starlings' aversion to eyespots as the basis for a novel 'cognitive bias' task? Applied Animal Behaviour Science 118: 182-190. http://dx.doi.org/10.1016/j.applanim.2009.02.015

Brydges NM, Hall H, Nicolson R, Holmes MC and Hall J 2012 The effects of juvenile stress on anxiety, cognitive bias and decision making in adulthood: a rat model. PLoS One 7: e48143. http://dx.doi.org/10.1371/journal.pone.0048143

Burgdorf J and Panksepp J 2006 The neurobiology of positive emotions. Neuroscience \& Biobehavioral Reviews 30: 173-187. http://dx.doi.org/10.1016/j.neubiorev.2005.06.001

Burman O, McGowan R, Mendl M, Norling Y, Paul E, Rehn T and Keeling L 2011 Using judgement bias to measure positive affective state in dogs. Applied Animal Behaviour Science 132: 160-168. http://dx.doi.org/10.1016/j.applanim.2011.04.001

Burman OHP, Parker RMA, Paul ES and Mendl MT 2008a A spatial judgement task to determine background emotional state in laboratory rats, Rattus norvegicus. Animal Behaviour 76: 801-809. http://dx.doi.org/10.1016/j.anbehav.2008.02.014 
Burman OHP, Parker RMA, Paul ES and Mendl MT 2008b Sensitivity to reward loss as an indicator of animal emotion and welfare. Biology Letters 4: 330-333. http://dx.doi.org/10.1098/rsbl.2008.0113

Burman OHP, Parker RMA, Paul ES and MendI MT 2009 Anxiety-induced cognitive bias in non-human animals. Physiology \& Behavior 98: 345-350. http://dx.doi.org/10.1016/j.physbeh.2009.06.012

Clore GL and Ortony A 2000 Cognition in emotion: always, sometimes or never? In: Lane RD and Nadel L (eds) Cognitive Neuroscience of Emotion pp. 24-61. Oxford University Press: Oxford, UK

Dantzer R 2002 Can farm animal welfare be understood without taking into account the issues of emotion and cognition? Journal of Animal Science 80: E1-E9

Daros RR, Costa JHC, von Keyserlingk MAG, Hötzel MJ and Weary DM 2014 Separation from the dam causes negative judgement bias in dairy calves. PLoS One 9: e98429. http://dx.doi.org/10.1371/journal.pone.0098429

Dawkins MS 2006 A user's guide to animal welfare science. Trends in Ecology and Evolution 25: 77-82. http://dx.doi.org/10.1016/j.tree.2005.10.017

Dawkins MS 2008 The science of animal suffering. Ethology 114: 937-945. http://dx.doi.org/10.1111/j.1439-0310.2008.01557.x

Department for Environment, Food and Rural Affairs 1989 Animal Welfare: Codes of Recommendations for the Welfare of Livestock - Goats. http://archive.defra.gov.uk/foodfarm/farmanimal/welfare/onfarm/ othersps/goatcode.htm

Desiré L, Boissy A and Veissier I 2002 Emotions in farm animals: a new approach to animal welfare in applied ethology. Behavioural Processes 60: 165-180

Destrez A, Deiss V, Belzung C, Lee C and Boissy A 2012 Does reduction of fearfulness tend to reduce pessimistic-like judgement in lambs. Applied Animal Behavioural Science 139: 233-241. http://dx.doi.org/10.1016/j.applanim.2012.04.006

Destrez A, Deiss V, Levy F, Calandreau L, Lee C, Chaillou-Sagon E and Boissy A 2013 Chronic stress induces pessimistic-like judgment and learning deficits in sheep. Applied Animal Behaviour Science 148: 28-36. http://dx.doi.org/10.1016/j.applanim.2013.07.016

Douglas C, Bateson M, Walsh C, Bédué A and Edwards SA 2012 Environmental enrichment induces optimistic cognitive biases in pigs. Applied Animal Behaviour Science 139: 65-73. http://dx.doi.org/10.1016/j.applanim.2012.02.018

Doyle RE, Fisher AD, Hinch GN, Boissy A and Lee C 2010 Release from restraint generates a positive judgement bias in sheep. Applied Animal Behaviour Science 122: 28-34. http://dx.doi.org/10.1016/j.applanim.2009.11.003

Doyle RE, Lee C, Deiss V, Fisher AD, Hinch GN and Boissy A 2011a Measuring judgement bias and emotional reactivity in sheep following long-term exposure to unpredictable and aversive events. Physiology \& Behavior 102: 503-510. http://dx.doi.org/10.1016/j.physbeh.2011.01.001

Doyle RE, Hinch GN, Fisher AD, Boissy A, Henshall JM and Lee C 2011b Administration of serotonin inhibitor p-Chlorophrnylalanine induces pessimistic-like judgment bias in sheep. Psychoneuroendocrinology 36: 279-288. http://dx.doi.org/10.1016/j.psyneuen.2010.07.018

Duncan IJH 1996 Animal welfare in terms of feelings. Acta Agriculturae Scandinavica 27: 29-35

Eysenck MW, Mogg K, May J, Richards A and Mathews A 1991 Bias in interpretation of ambiguous sentences related to threat and anxiety. Journal of Abnormal Psychology 100: 144-150. http://dx.doi.org/10.1037/0021-843X.100.2.144

FAWC 2013 Annual review 2012-2013. www.defra.gov.uk/fawc/files/Annual-Review-2012-2013.pdf

Gotlib IH and Krasnoperova E 1998 Biased information processing as a vulnerability factor in depression. Behavior Therapy 29: 603-617. http://dx.doi.org/10.1016/S0005-7894(98)80020-8

Harding EJ, Paul ES and MendI M 2004 Animal behaviour: cognitive bias and affective state. Nature 427: 312. http://dx.doi.org/10.1038/427312a 
Haselton MG and Nettle D 2006 The paranoid optimist: an integrative evolutionary model of cognitive biases. Personality and Social Psychology Review 10: 47-66. http://dx.doi.org/10.1207/s15327957pspr1001_3

Johnson E and Tversky A 1983 Affect, generalization, and the perception of risk. Journal of Personality and Social Psychology 45: 20-31. http://dx.doi.org/10.1037/0022-3514.45.1.20

Keen HA, Nelson OL, Robbins CT, Evans M, Shepherdson DJ and Newberry RC 2013 Validation of a novel cognitive bias task based on difference in quantity of reinforcement for assessing environmental enrichment. Animal Cognition 17: 529-541. http://dx.doi.org/10.1007/s10071-0130684-1

Lerner JS and Keltner D 2000 Beyond valence: toward a model of emotion-specific influences on judgement and choice. Cognition \& Emotion 14: 473-493. http://dx.doi.org/10.1080/026999300402763

MacLeod C, Mathews A and Tata P 1986 Attentional bias in emotional disorders. Journal of Abnormal Psychology 95: 15-20. http://dx.doi.org/10.1037/0021-843X.95.1.15

Matheson SM, Asher L and Bateson M 2008 Larger enriched cages are associated with "optimistic" response biases in captive European starlings (Sturnus vulgaris). Applied Animal Behaviour Science 109: 374-383. http://dx.doi.org/10.1016/j.applanim.2007.03.007

Mathews A and MacLeod C 1985 Selective processing of threat cues in anxiety states. Behaviour Research and Therapy 23: 563-569. http://dx.doi.org/10.1016/0005-7967(85)90104-4

Mathews A and MacLeod C 1994 Cognitive approaches to emotion and emotional disorders. Annual Review of Psychology 45: 25-50. http://dx.doi.org/10.1146/annurev.ps.45.020194.000325

Mendl M, Brooks J, Basse C, Burman O, Paul E, Blackwell E and Casey R 2010b Dogs showing separation-related behavior exhibit a pessimistic cognitive bias. Current Biology 20: R839-R840. http://dx.doi.org/10.1016/j.cub.2010.08.030

Mendl M, Burman OHP, Parker R and Paul E 2009 Cognitive bias as an indicator of animal emotion and welfare: emerging evidence and underlying mechanisms. Applied Animal Behaviour Science 118: 161-181. http://dx.doi.org/10.1016/j.applanim.2009.02.023

MendI M, Burman OHP and Paul ES 2010a An integrative and functional framework for the study of animal emotion and mood. Proceedings of the Royal Society B277: 2895-2904. http://dx.doi.org/10.1098/rspb.2010.0303

Mineka S, Watson D and Clark LA 1998 Comorbidity of anxiety and unipolar mood disorders. Annual Review of Psychology 49: 377-412. http://dx.doi.org/10.1146/annurev.psych.49.1.377

Moher D, Liberati A, Tetzlaff J, Altman DG and The PRISMA Group 2009 Preferred reporting items for systematic reviews and meta-analyses: The PRISMA Statement. PLoS Medicine 6: e1000097. http://dx.doi.org/10.1371/journal.pmed.1000097

Neave HW, Daros RR, Costa JHC, von Keyserlingk MAG and Weary DM 2013 Pain and pessimism: dairy calves exhibit negative judgement bias following hot-iron disbudding. PLoS One 8:e80556. http://dx.doi.org/10.1371/journal.pone.0080556

Nettle D and Bateson M 2012 The evolutionary origins of mood and its disorders. Current Biology 22: R712-R721. http://dx.doi.org/10.1016/j.cub.2012.06.020

Nygren TE, Isen AM, Taylor PJ and Dulin J 1996 The influence of positive affect on the decision rule in risky situations. Organizational Behavior and Human Decision Processes 66: 59-72. http://dx.doi.org/10.1006/obhd.1996.0038

Panksepp J 2005 Affective consciousness: core emotional feelings in animals and humans. Consciousness and Cognition 14: 30-80. http://dx.doi.org/10.1016/j.concog.2004.10.004

Papciak J, Popik P, Fuchs E and Rygula R 2013 Chronic psychosocial stress makes rats more 'pessimistic' in the ambiguous cue interpretation paradigm. Behavioural Brain Research 256: 305310. http://dx.doi.org/10.1016/j.bbr.2013.08.036 
Paul ES, Harding EJ and Mendl M 2005 Measuring emotional processes in animals: the utility of a cognitive approach. Neuroscience and Biobehavioral Reviews 29: 469-491. http://dx.doi.org/10.1016/j.neubiorev.2005.01.002

Pomerantz O, Terkel J, Suomi SJ and Paukner A 2012 Stereotypic head twirls, but not pacing, are related to a 'pessimistic'-like judgment bias among captive tufted capuchins (Cebus apella). Animal Cognition 15: 689-698. http://dx.doi.org/10.1007/s10071-012-0497-7

Richter S, Schick A, Hoyer C, Lankisch K, Gass P and Vollmayr B 2012 A glass full of optimism: enrichment effects on cognitive bias in a rat model of depression. Cognitive, Affective \& Behavioral Neuroscience 12: 527-542. http://dx.doi.org/10.3758/s13415-012-0101-2

Rolls ET 2005 Emotion Explained. Oxford University Press: Oxford, UK. http://dx.doi.org/10.1093/acprof:oso/9780198570035.001.0001

Russell JA 2003 Core affect and the psychological construction of emotion. Psychological Review 110: 145-172. http://dx.doi.org/10.1037/0033-295X.110.1.145

Rygula R, Pluta H and Popik P 2012 Laughing rats are optimistic. PLoS One 7: e51959. http://dx.doi.org/10.1371/journal.pone.0051959

Salmeto AL, Hymel KA, Carpenter EC, Brilot BO, Bateson M and Sufka KJ 2011 Cognitive bias in the chick anxiety-depression model. Brain Research 1373: 124-130. http://dx.doi.org/10.1016/j.brainres.2010.12.007

Sandem Al, Janczak AM, Salte R and Braastad BO 2006 The use of diazepam as a pharmacological validation of eye white as an indicator of emotional state in dairy cows. Applied Animal Behaviour Science 96: 177-183. http://dx.doi.org/10.1016/j.applanim.2005.06.008

Sanger ME, Doyle RE, Hinch GN and Lee C 2011 Sheep exhibit a positive judgement bias and stressinduced hyperthermia following shearing. Applied Animal Behaviour Science 131: 94-103. http://dx.doi.org/10.1016/j.applanim.2011.02.001

Spruijt BM, van den Bos R and Pijlman FTA 2001 A concept of welfare based on reward evaluating mechanisms in the brain: anticipatory behaviour as an indicator for the state of reward systems. Applied Animal Behaviour Science 72: 145-171. http://dx.doi.org/10.1016/S0168-1591(00)002045

Trimmer PC, Paul ES, MendI MT, McNamara JM and Houston AI 2013 On the evolution and optimality of mood states. Behavioural Sciences 3: 501-521. http://dx.doi.org /10.3390/bs3030501

Verbeek E, Ferguson D and Lee C 2014a Are hungry sheep more pessimistic? The effects of food restriction on cognitive bias and the involvement of ghrelin in its regulation. Physiology \& Behaviour 123: 67-75. http://dx.doi.org/10.1016/j.physbeh.2013.09.017

Verbeek E, Ferguson D, de Monjour PQ and Lee C 2014b Generating positive affective state in sheep: the influence of food rewards and opioid administration. Applied Animal Behavior Science 154: 39-47. http://dx.doi.org/10.1016/j.applanim.2014.02.011

Wathes C 2010 Lives worth living? Veterinary Record 166: 468-469. http://dx.doi.org/10.1136/vr.c849

Wichman A, Keeling LJ and Forkman B 2012 Cognitive bias and anticipatory behaviour of laying hens housed in basic and enriched pens. Applied Animal Behaviour Science 140: 62-69. http://dx.doi.org/10.1016/j.applanim.2012.05.006

Wright WF and Bower GH 1992 Mood effects on subjective probability assessment. Organizational Behavior and Human Decision Processes 52: 27. http://dx.doi.org/10.1016/0749-5978(92)90039A 